\author{
Josef G. Heckmann \\ Christoph J.G. Lang \\ Margarete Weber \\ Bernd Tomandl \\ Bernhard Neundörfer
}

\section{Migraine-like headache as the presenting symptom of basilar artery occlusion}

Received: 19 August 2002

Accepted in revised form: 2 December 2002

J.G. Heckmann (西) • C.J.G. Lang

M. Weber • B. Neundörfer

Department of Neurology,

University of Erlangen-Nuremberg,

Schwabachanlage 6,

D-91054 Erlangen, Germany

e-mail: josef.heckmann@neuro.imed.uni-

erlangen.de

Tel.: +49-9131-8534457

Fax: +49-9131-8534436

B. Tomandl

Department of Neuroradiology,

University of Erlanger-Nuremberg,

Erlangen, Germany

\begin{abstract}
If migraine or a migrainelike headache and stroke occur together, it is difficult to determine whether migraine is the cause of the stroke or stroke is the cause of symptomatic migraine. We report the case of a 19-year-old woman without a history of migraine who presented with a migraine-like headache, nausea and desire for tranquility and dimmed lighting. Initial neurological examination, computed tomography and cerebrospinal fluid analysis were normal, leading to the presumptive diagnosis of first manifestation of migraine. Persistence of dizziness and transient diplopia, however, prompted a mag-
\end{abstract}

netic resonance imaging examination, which revealed major stroke in the posterior circulation due to occlusion of the basilar artery. The symptoms resolved spontaneously and treatment with antiplatelet inhibitor was prescribed. Smoking and use of oral contraceptives were identified as vascular risk factors. Stroke in the posterior circulation due to occlusion of the basilar artery may show rather inconspicuous symptoms and provoke migrainous headache.

Key-words Migraine $\cdot$ Headache Stroke $\cdot$ Basilar occlusion

\section{Introduction}

If migraine or a migraine-like headache syndrome and stroke occur together, it is difficult to determine whether migraine is the cause of the stroke or stroke is the cause of symptomatic migraine [1]. We report the case of a 19-year-old woman without a history of migraine who presented with a migraine-like headache due to major stroke in the posterior circulation. The stroke due to occlusion of the basilar artery is discussed to have provoked the headache attack.

\section{Case report}

A 19-year-old woman woke up in the morning with a throbbing headache in the neck and vomited. She felt dizzy but was able to go to her job as a hotel manager. There she reported a persisting headache, predominantly in the neck, nausea and desire for tranquility and dimmed lighting, finally leading to admission at our department. On admission she was fully oriented without focal neurological signs. Neither cranial computed tomography (CT) or analysis of cerebrospinal fluid (CSF) showed anything unusual. She was treated with ibuprofen (600 $\mathrm{mg}$ b.i.d.) and metoclopramide ( $8 \mathrm{mg}$ orally) and was discharged. The presumptive diagnosis was first manifestation of migraine. However, the dizziness did not completely disappear and new diplopia occurred five days later and lasted two days, leading to a magnetic resonance imaging (MRI) examination which now revealed right-sided cerebellar infarction and occlusion of the basilar artery slightly above the anterior inferior cerebellar artery and below the superior cerebellar artery. The right posterior inferior cerebellar artery could not be visualized (Figs. 1, 2). 


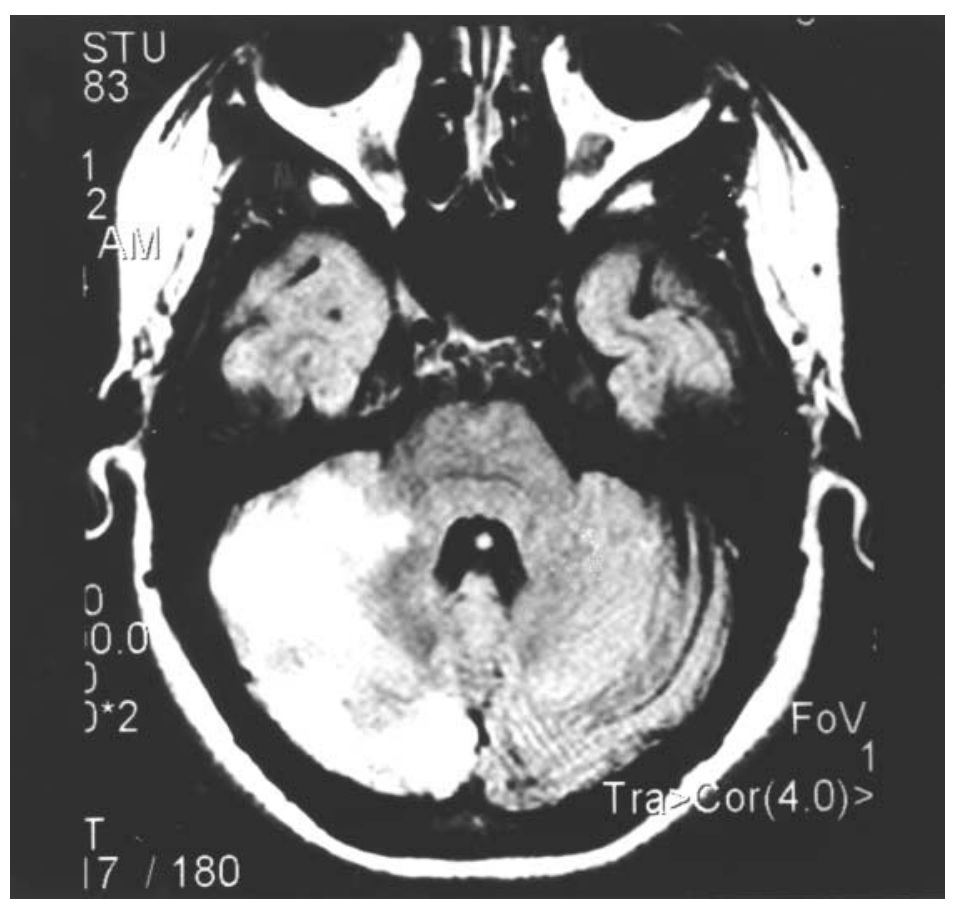

Fig. 1 MR image (FLAIR technique) obtained 5 days after the inital symptoms demonstrated an extensive right-sided cerebellar lesion concordant with acute ischemic stroke

Fig. 2 MRI angiography (lateral view rotated $20^{\circ}$ ) demonstrated occlusion of the basilar artery slightly above the branch of the anterior inferior cerebellar artery and below the superior cerebellar artery. The right posterior inferior cerebellar artery could not be visualized

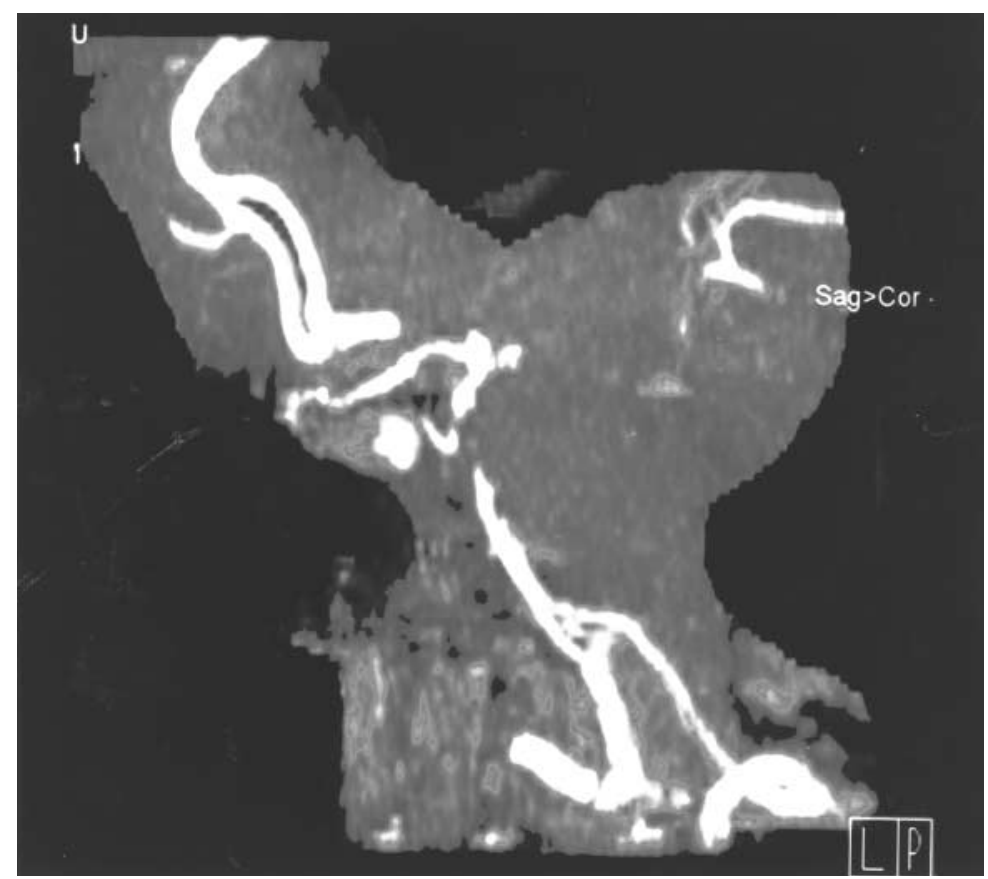

On readmission, the patient was free from neurological signs and symptoms. Treatment using thrombolytic agents was not indicated at this time. Thus, antiplatelet inhibitor acetylsalicylic acid (250 mg per day) was prescribed. An extensive work-up including transthoracic echocardiography, contrast transcranial Doppler sonography and thrombophilia screening showed nothing extraordinary. In particular, there was no reason to suspect a patent foramen ovale. Smoking and use of oral contraceptives were identified as cerebrovascular risk factors. At follow-up four months later, the patient was free of symptoms and MR angiography was unchanged. The patient stopped smoking and the throbbing headache did not recur. 


\section{Discussion}

Occlusion of the basilar artery is still a serious disease with a high mortality rate. Clinically, patients typically have impaired consciousness, oculomotor disturbances, diplopia, vertigo, dysarthria, cerebellar ataxia and often bilateral motor deficits including Babinski's signs. Headache has been reported to occur in about $25 \%$ of cases but it is neither the major nor the leading symptom [2]. Early diagnosis is mandatory to apply modern therapeutic methods such as systemic or local fibrinolysis with rtPA, treatment with abciximab or interventional neuroradiological methods including local stenting [3]. In our patient the initial prominent signs and symptoms were awakening with throbbing headache, dizziness, nausea, vomiting and phono- and photophobia. In the absence of focal neurological signs, a first attack of migraine without aura was suspected in accordance with the diagnostic criteria for migraine according to the International Headache Society classification [4]. Serious meningitis and subarachnoidal hemorrhage were excluded by brain CT and lumbar puncture. In addition, the rapid clinical improvement after administration of ibuprofen and metoclopramide [5] supported this assumption. Thus, the patient was sent home without further investigation. However, five days later, the patient reported transient diplopia prompting an ancillary MRI examination, which detected cerebellar infarction and occlusion of the basilar artery.

Retrospectively, we believe that, in our patient, the migraine-like headache was induced by ischemia. A migraine-associated stroke seems improbable because the patient did not report former migraine attacks and there were no recurring migraine attacks afterwards.

The topic of ischemia-induced (symptomatic) migraine is still a matter of debate. Clinical experience suggests that the situation of ischemia-induced migraine attacks may be more frequent than migraine-induced ischemic insults $[1,6$, 7]. In acute stroke, a number of biochemical changes occur which seem to have the potential to provoke a migraine attack in susceptible individuals. For instance NO (nitric oxide) or glutamate which are elevated in cerebral ischemia play a major role in the pathophysiology of headache occuring in both stroke and migraine [8, 9]. Additionally, a number of the inflammatory mediators that arise in ischemic areas are able to provoke headache [10]. Furthermore, it is known that various vascular diseases can provoke headache or even migraine, such as moyamoya disease, Raynaud's syndrome, Sneddon's syndrome, CADASIL, ergotism, giant cell arteritis, cerebral vasculitis, fibromuscular dysplasia and cerebral venous sinus thrombosis [11-13]. In particular in our patient with ischemia in the posterior circulation, it seems not implausible that the complex ischemic condition activated the trigeminovascular system, thus provoking a migrainous headache [14].

We feel that the reported case is interesting for two reasons. First, basilar occlusion with prominent cerebellar infarction did initially show rather inconspicuous symptoms and no noticeable neurological signs. It was not until five days later that the patient suffered first focal neurological signs in the form of diplopia, probably due to local swelling in the posterior fossa, and thus promping a second evaluation. Second, in patients with a first-ever migraine-like headache syndrome, in particular if mimicking a migraine attack, the differential diagnosis should include stroke, imminent in the posterior circulation. Based on this experience we propose to perform a thorough neurovascular workup. Besides neurosonographic methods including transcranial Doppler sonography, modern MRI techniques will allow diagnosis of hyperacute stroke and thus the application of modern treatment regimens if necessary [15].

\section{References}

1. Olesen J, Friberg L, Olsen TS, Andersen AR, Lassen NA, Hansen PE, Karle A (1993) Ischaemia-induced (symptomatic) migraine attacks may be more frequent than migraineinduced ischaemic insults. Brain 116:187-202

2. Devuyst G, Bogousslavsky J, Meuli R, Moncayo J, de Freitas G, van Melle G (2002) Stroke or transient ischemic attacks with basilar artery stenosis or occlusion: clinical patterns and outcome. Arch Neurol 59:567-573
3. Eckert B, Koch C, Thomalla G, Roether J, Zeumer H (2002) Acute basilar artery occlusion treated with combined intravenous abciximab and intra-arterial tissue plasminogen activator: report of 3 cases. Stroke 33:1424-1427

4. - (1988) Classification and diagnostic criteria for headache disorders, cranial neuralgias and facial pain. Headache Classification Committee of the International Headache Society. Cephalalgia 8[Suppl 7]:1-96
5. Diener HC, Limmroth V (2001) Advances in pharmacological treatment of migraine. Expert Opin Investig Drugs 10:1831-1845

6. Heckmann JG, Lang CJ, Neundörfer B (2002) Ischemic stroke and active migraine. Neurology 59:149-150

7. Heckmann JG, Lang CJ, Dietrich W, Neidhardt B, Neundörfer B (2002) Symptomatic migraine linked to stroke due to paradoxical embolism and elevated thrombosis risk. Cephalalgia 22:154-156

8. Bredt DS (1999) Endogenous nitric oxide synthesis: biological functions and pathophysiology. Free Radic Res 31:577-596 
9. Castillo J, Martinez F, Corredera E, Aldrey JM, Noya M (1995) Amino acid transmitters in patients with headache during the acute phase of cerebrovascular ischemic disease. Stroke 26:2035-2039

10. Leira R, Davalos A, Aneiros A, Serena J, Pumar JM, Castillo J (2002) Headache as a surrogate marker of the molecular mechanisms implicated in progressing stroke. Cephalalgia 22:303-308
11. Hosain SA, Hughes JT, Forem SL, Wisoff J, Fish I (1994) Use of a calcium channel blocker (nicardipine $\mathrm{HCl}$ ) in the treatment of childhood moyamoya disease. J Child Neurol 9:378-380

12. Heckmann JG, Tomandl B, Kraus B, Gerlach R, Neundörfer B (2000) A case of diffuse cerebral vasospasmpossibly ergotamine-associated? Cerebrovasc Dis 10:417-418

13. Slooter AJC, Ramos LMP, Kappelle LJ (2002) Migraine-like headache as the presenting symptom of cerebral venous sinus thrombosis. J Neurol 249:775-776
14. Weiller C, May A, Limmroth V, Jüptner M, Kaube H, van Schayck RV, Coenen HH, Diener HC (1995) Brainstem activation in spontaneous human migraine attacks. Nat Med 1:658-660

15. Fiebach JB, Schellinger PD (2002) Modern nuclear magnetic resonance techniques in stroke. Nervenarzt 73:104-116 (in German) 